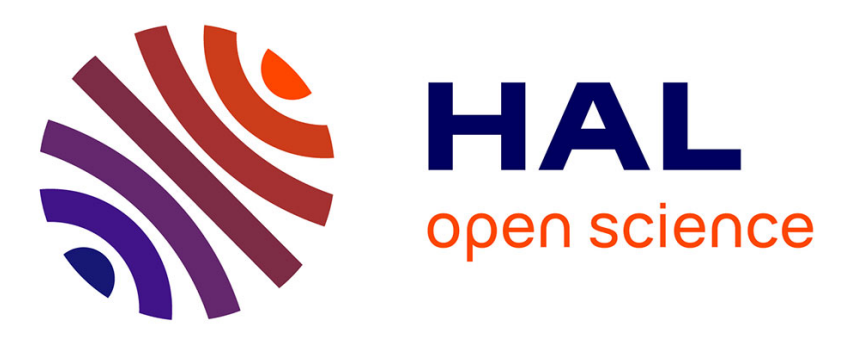

\title{
Solving the Inclination Sign Ambiguity in Three Dimensional Polarized Light Imaging with a PDE-Based Method
}

\author{
Abib O. Y. Alimi, Marco Pizzolato, Rutger H.J. Fick, Rachid Deriche
}

\section{- To cite this version:}

Abib O. Y. Alimi, Marco Pizzolato, Rutger H.J. Fick, Rachid Deriche. Solving the Inclination Sign Ambiguity in Three Dimensional Polarized Light Imaging with a PDE-Based Method. ISBI 2017 - IEEE International Symposium on Biomedical Imaging, Apr 2017, Melbourne, Australia. hal01533363

\section{HAL Id: hal-01533363 \\ https://hal.inria.fr/hal-01533363}

Submitted on 6 Jun 2017

HAL is a multi-disciplinary open access archive for the deposit and dissemination of scientific research documents, whether they are published or not. The documents may come from teaching and research institutions in France or abroad, or from public or private research centers.
L'archive ouverte pluridisciplinaire HAL, est destinée au dépôt et à la diffusion de documents scientifiques de niveau recherche, publiés ou non, émanant des établissements d'enseignement et de recherche français ou étrangers, des laboratoires publics ou privés. 


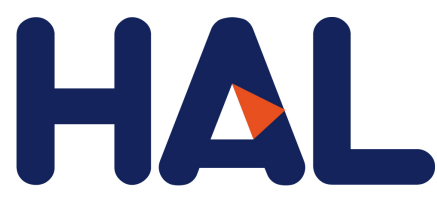

archives-ouvertes

\title{
Solving the Inclination Sign Ambiguity in Three Dimensional Polarized Light Imaging with a PDE-Based Method
}

\author{
Abib O. Y. Alimi, Marco Pizzolato, Rutger Fick, Rachid Deriche
}

\section{To cite this version:}

Abib O. Y. Alimi, Marco Pizzolato, Rutger Fick, Rachid Deriche. Solving the Inclination Sign Ambiguity in Three Dimensional Polarized Light Imaging with a PDE-Based Method. ISBI 2017 - IEEE International Symposium on Biomedical Imaging, Apr 2017, Melbourne, Australia. <hal-01533363v2>

HAL Id: hal-01533363

https://hal.inria.fr/hal-01533363v2

Submitted on 6 Mar 2018

HAL is a multi-disciplinary open access archive for the deposit and dissemination of scientific research documents, whether they are published or not. The documents may come from teaching and research institutions in France or abroad, or from public or private research centers.
L'archive ouverte pluridisciplinaire $\mathbf{H A L}$, est destinée au dépôt et à la diffusion de documents scientifiques de niveau recherche, publiés ou non, émanant des établissements d'enseignement et de recherche français ou étrangers, des laboratoires publics ou privés. 


\title{
Solving the Inclination Sign Ambiguity in Three Dimensional Polarized Light Imaging with a PDE-Based Method
}

\author{
Abib Alimi $\quad$ Marco Pizzolato ${ }^{\dagger} \quad$ Rutger H.J. Fick Rachid Deriche \\ Université Côte d'Azur, Inria, France
}

\begin{abstract}
Three dimensional Polarized Light Imaging (3D-PLI) is a contrast-enhancing technique that measures the spatial fiber architecture in the human postmortem brain or heart at a submillimeter resolution. In a voxel, the 3D fiber orientation is defined by the direction angle and the inclination angle whose sign is unknown. To have an accurate explanation of fiber orientation, it is compulsory to clear up this sign ambiguity. A tilting process provides information about the true inclination sign, however the technique is highly sensitive to noise. In this work, a partial differential equations based method is proposed to reduce the noise: the total variation model of Rudin-Osher-Fatemi is extended to 3D orientation vector images to restore the sign. The proposed algorithm is evaluated on synthetic and human heart data and results show that the true sign of the inclination angle can be successfully extracted.
\end{abstract}

Index Terms - PLI, total variation, PDE, 3D vector image

\section{INTRODUCTION}

To understand and cure cardiac and brain disorders, it is fundamental to investigate the function as well as the structural architecture of the heart and the brain. 3D-PLI is an optical approach that utilizes the birefringence in these postmortem organs to map their spatial fibers structure with a resolution at the submillimeter scale.

3D Polarized light imaging: The polarimeter, which is a 3D-PLI experimental setup, is optimized for high-resolution imaging of unstained histological sections. Generally, it consists of a light source, a linear polarizer, a tiltable specimen stage holding the tissue section, a quarter-wave retarder, a second linear polarizer called analyzer, and a camera, see Figure 2A. A series of intensity images is then produced as the polarizing filters are simultaneously rotated.

Linearly polarized light passes through the sample and allows to detect its birefringent structure. The outgoing light permits to describe the spatial fiber orientation by a pair of angles, see Figure 2B:

\footnotetext{
${ }^{\dagger}$ The author expresses his thanks to Olea Medical and to the ProvenceAlpes-Côte d'Azur Regional Council for providing grant and support.
}

- the in-plane or direction angle $\varphi$ determining the $\mathrm{x}-\mathrm{y}$ orientation of the fiber;

- the out-of-plane or inclination angle $\alpha$, the vertical component of the fiber.

Therefore, as mentioned in [1], each histological section is characterized by a 3D vector field visualized as a fiber orientation map (FOM), see Figure 1. FOMs show the dominant fiber orientation in each voxel.

The fiber orientation vector $v_{i}$ at voxel location $i$ is parameterized by the two angles $\varphi_{i}$ and $\alpha_{i}$ as follows:

$$
v_{i}=\left(\begin{array}{c}
\cos \alpha_{i} \cdot \cos \varphi_{i} \\
\cos \alpha_{i} \cdot \sin \varphi_{i} \\
\sin \alpha_{i}
\end{array}\right)
$$

The voxel dimensions are defined by the image pixel size and the section thickness.

The angles $\varphi$ and $\alpha$ are restricted to $\left[0^{\circ}, 180^{\circ}\right]$ and $\left[-90^{\circ}, 90^{\circ}\right]$ respectively. The inclination angle $\alpha$ can be derived from equation (6) in [1], however it has a nonlinear relation with $r$ which is the retardation parameter, and can be approximated as

$$
|\alpha|=\arccos \left(\sqrt{\frac{2 \cdot \arcsin (r)}{\pi \cdot d_{\text {rel }}}}\right)
$$

where $d_{r e l}$ is a reference value or relative thickness depending on the section thickness, the birefringence and the wavelength. Equation (2) gives only the absolute value of $\alpha$, which introduces the inclination sign ambiguity in 3D-PLI. It is compulsory to clear up this ambiguity in order to have a correct spatial fiber orientation in each voxel. Indeed, 3DPLI based tractography can then be performed and utilized as a validator of tractography based on diffusion magnetic resonance imaging.

To address this sign ambiguity in microscope imaging, $\mathrm{Pa}-$ jdzik et al. [2] developed a tilting-stage to discriminate positive and negative inclination signs of crystal orientations. A similar tilting setup is built for 3D-PLI by [3], as displayed in Figure 2A.

Tilting setup: Measurements from the tiltable specimen stage give additional information about the inclination sign at each individual pixel. The stage can be tilted in two axes, i-e 


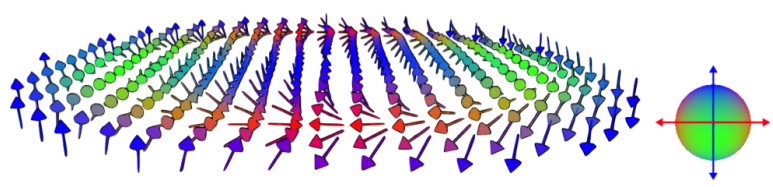

Fig. 1. An example of simulated FOM enabling a clear vector visualization of the $3 \mathrm{D}$ fiber orientation vectors in a histological tissue slice. Adapted from [4]

four geographical directions $\psi \in\left\{N=90^{\circ}, W=180^{\circ}, S=\right.$ $\left.270^{\circ}, E=0^{\circ}\right\}$ : the edge of the specimen in the specified direction $\psi$ is tilted down towards the light source by an angle $\tau \approx 4^{\circ}$ to avoid strong distortions.

The sign of $\alpha$ can be determined most accurately when $\varphi$ and $\psi$ are similar: positively inclined fibers can be discriminated from negatively inclined ones by their decrease in absolute inclination after tilting. Otherwise, the change is less important or even inverted. Dohmen [4] noticed that these changes are marginal and hence very sensitive to noise and proposed this signun function which describes this relationship as

$$
\operatorname{sgn}(\alpha)=\operatorname{sgn}\left(\left|\alpha^{\psi \pm 180^{\circ}}\right|-\left|\alpha^{\psi}\right|\right) \cdot \operatorname{sgn}(\cos (\varphi-\psi))
$$

where $\alpha^{\psi}$ denotes the tilted measurement for each tilting direction $\psi$ and $\psi \pm 180^{\circ}$ which is the opposite direction.

Measurements from the tiltable specimen stage allow to derive the sign of the inclination angle but it is highly sensitive to noise. In this work, the total variation (TV) model of Rudin-Osher-Fatemi (ROF) is extended to 3D vector images and proposed for noise removal and restoration of the proper sign of the in-plan angle.

\section{INCLINATION SIGN RESTORATION}

To correct the wrong inclination signs, a partial differential equations (PDEs) method, which has been widely used over the past decades with edge preservation, is considered. This is interesting because different fiber populations located within a region seem to be delimited by some edges at their intersections.

The restoration of the sign of $\alpha$ is performed via a PDE method which is based on a variational approach of energy functional minimization: the total variation (TV) model of Rudin-Osher-Fatemi (ROF) [5]. In this work, the ROF model is extended and applied to tackle our problem of restoring 3D orientation vectors images.

\subsection{Rudin-Osher-Fatemi model}

The total variation minimizing process of ROF is a classical variational denoising algorithm developed for scalar images. This algorithm looks for an equilibrium state of an energy
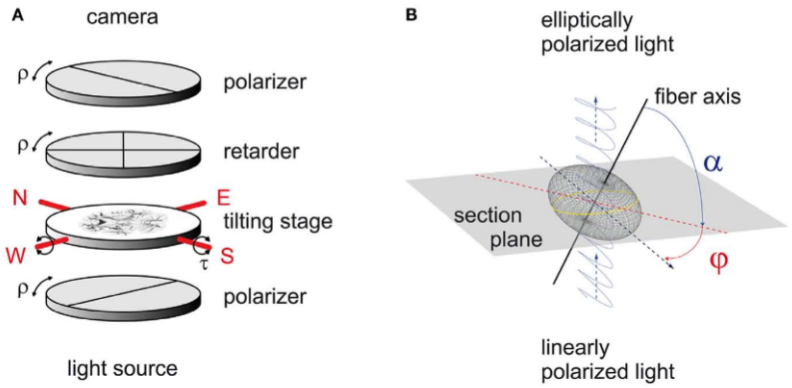

Fig. 2. Basic principles of 3D-PLI. (A) Polarimeter setup with tilting stage. (B) Optical fiber model. Image from [3]

functional which consists of the TV norm of the image $I$ and the fidelity of the image to the noisy input image $I_{0}$.

The idea is to find the (unique) minimizer, $I$, of

$$
\min _{I} E(I)=\frac{\lambda}{2} \int_{\Omega}\left|I_{0}-I\right|^{2} d x d y+\int_{\Omega}|\nabla I| d x d y,
$$

with $\lambda>0$ is a scaling parameter controlling the fidelity of the solution to the input image.This is further generalized by the $\Phi$-formulation [6] of the functional whose associated Euler-Lagrange $(E L)$ equation is

$$
-F \equiv \lambda\left(I_{0}-I\right)+\operatorname{div}\left(\Phi^{\prime}(\cdot) \frac{\nabla I}{|\nabla I|}\right)=0
$$

where Neumann boundary conditions are assumed. The solution is usually found by

$$
I_{t}=-F, \quad \text { and }\left.\quad I\right|_{t=0}=I_{0}
$$

The actual $\Phi$ function used in this work is $\Phi(x)=$ $\sqrt{\epsilon^{2}+x^{2}}$ with $\epsilon>0$ a small parameter. This removes the singularity when $|\nabla I|=0$ by approximating $E(I)$ and allows an easy implementation by standard discretization of the Euler-Lagrange equations.

In the following sections, the ROF model is adapted and applied to restore the inclination angle sign in pixels where the tilting process is not efficient enough. The aim is now to perform the TV minimizing process on 3D fiber orientation vectors images for denoising and finding the sign of $\alpha$ for a given histological FOM.

Consequently, the finally signed inclination angle $\alpha$ is obtained by multiplication of the absolute angle with the sign of the restored angle $\alpha_{\text {rof }}$, as follow:

$$
\alpha=|\alpha| \cdot \operatorname{sgn}\left(\alpha_{\text {rof }}\right)
$$

\subsection{ROF on sectional fiber orientation map}

Method: Let us recall that in 3D-PLI each histological section is characterized by a $3 \mathrm{D}$ vector field visualized as a fiber orientation map (FOM) indicating the prevailing fiber orientation in each voxel [1]. The TV process is extended to 3D 
and executed to denoise the FOM (built from the noisy tilting measurements) in order to derive the inclination angle sign. The functional to be minimized is

$$
\begin{aligned}
E(V)= & \frac{\lambda}{2} \int_{\Omega}\left|V_{0}-V\right|^{2} d x d y d z \\
& +\int_{\Omega}|\nabla V| d x d y d z+\gamma\left(|V|^{2}-1\right)
\end{aligned}
$$

where the third term, which is controlled by the parameter $\gamma$, constrains the recovered fiber orientation vector in a unit sphere, i-e $|V|=\sqrt{u^{2}+v^{2}+w^{2}}=1$ is its unit norm, $V_{0}$ is the noisy fiber orientation vector computed from the tilting process using equation (1), $V$ is the reconstructed vector given by the ROF minimization of $E$ and $u, v$ and $w$ are its components.

The associated Euler-Lagrange equations are

$$
-\lambda\left(V_{0}-V\right)-\operatorname{div}\left(\Phi^{\prime}(|\nabla V|) \frac{\nabla V}{|\nabla V|}\right)+2 \gamma V=0 \text { in } \Omega,
$$

with $\frac{\partial V}{\partial n}=0 \quad$ on the boundary of $\Omega=\partial \Omega$.

Note that, to $u, v$ and $w$, are associated the respective Euler-Lagrange equations $E L_{u}, E L_{v}$ and $E L_{w}$. The value of $\gamma$ is thus determined by multiplying each component to its corresponding EL equation, to obtain $u \times E L_{u}, v \times E L_{v}$ and $w \times E L_{w}$ which are summed and simplified to give

$$
\gamma=\frac{1}{2} \sum_{i=1}^{3} \operatorname{div}\left(\Phi^{\prime}\left(\left|\nabla v_{i}\right|\right) \frac{\nabla v_{i}}{\left|\nabla v_{i}\right|}\right)+\frac{\lambda}{2}\left(\sum_{i=1}^{3} v_{i}\left(v_{0}\right)_{i}-1\right)
$$

$\gamma$ is then injected in the Euler-Lagrange equations and the solution is found by simultaneously solving

$$
\begin{aligned}
u_{t}= & \lambda\left[v\left(v u_{0}-u v_{0}\right)+w\left(w u_{0}-u w_{0}\right)\right] \\
& -u\left[v \operatorname{div}\left(\Phi^{\prime}(|\nabla v|) \frac{\nabla v}{|\nabla v|}\right)+w \operatorname{div}\left(\Phi^{\prime}(|\nabla w|) \frac{\nabla w}{|\nabla w|}\right)\right] \\
& +\left(1-u^{2}\right) \operatorname{div}\left(\Phi^{\prime}(|\nabla u|) \frac{\nabla u}{|\nabla u|}\right)
\end{aligned}
$$

for component $u$, and $v_{t}, w_{t}$ for $v$ and $w$ respectively. The sign of $\alpha$ is the same as the sign the vertical component $(w)$ of the restored 3D fiber orientation vector.

Results on synthetic data: The extended ROF algorithm is evaluated on a synthetic histological FOM where orientation vectors are computed from synthetic $\varphi$ and $\alpha$ maps chosen so as to have four fiber populations with different orientations. Then noise is simulated and added to the angles to create noisy orientation vectors.

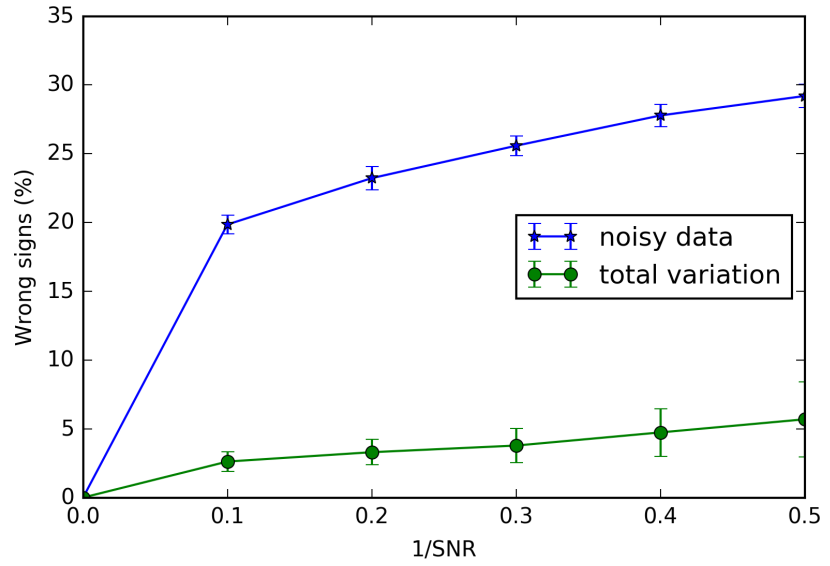

Fig. 3. Percentage of wrongly estimated inclination signs as noise increases. Each data point corresponds to the mean and standard deviation over 100 noisy realizations.

The algorithm is performed on the noisy FOM ( $n F O M$ ) to produce new orientation vectors, $t v F O M$. The idea is then to compare the amount of wrong inclination signs from $n F O M$ to those obtained after restoration. In Figure 3, results show that with different signal-to-noise ratios (SNR), the percentage of wrong signs in $n F O M$ is highly reduced after restoration. This indicates the algorithm significantly reduces the wrong signs and therefore clears up the ambiguity.

Results on human heart data: The human data consist of PLI measurements of histological sections of a whole cardiac myocardium. The absolute inclination angle map of a ventricular slice is chosen as shown in Figure 4A. The tilting process is performed and the corresponding $\alpha$ map is displayed in Figure 4B where the wrongly estimated signs are easily visible almost everywhere on the tissue when referring to the color map.

The extended TV minimization is then applied to the noisy FOM built from the tilting measurements and the result is shown in Figure 4C. Here, one can see some wrong signs but the ROF process has crucially restored them. This improvement is more observable on the zoomed region of interest in Figures 4D and 4E where the fibers of the left and right ventricles meet the fibers of the septum.

To quantify the effect of our TV algorithm on the real data, the randomness of signs of $\alpha$ from the tilting measurements and the TV process has been investigated. A local entropy filter with a radius of 5 is therefore applied to both inclination maps, then the mean entropy is computed and a reduction of $46 \%$ of the randomness of signs is achieved by the extended TV process as illustrated in Figures 4B and 4C.

\section{DISCUSSION AND CONCLUSION}

In this work, the proposed TV model is evaluated on both synthetic and human cardiac data and results reveal that the 


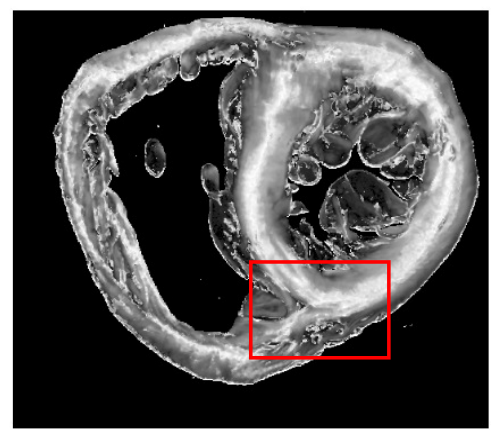

A
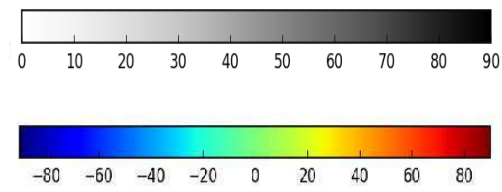

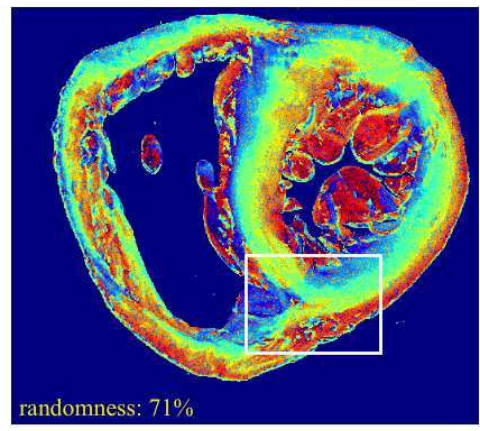

B

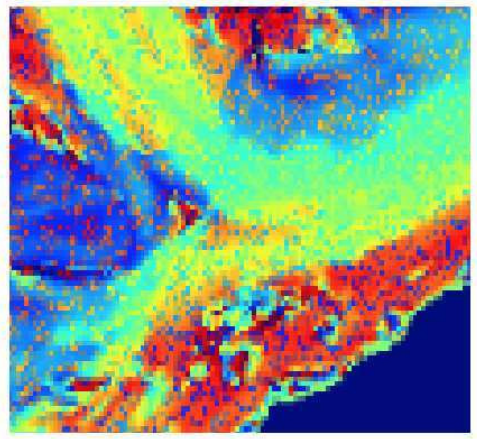

D

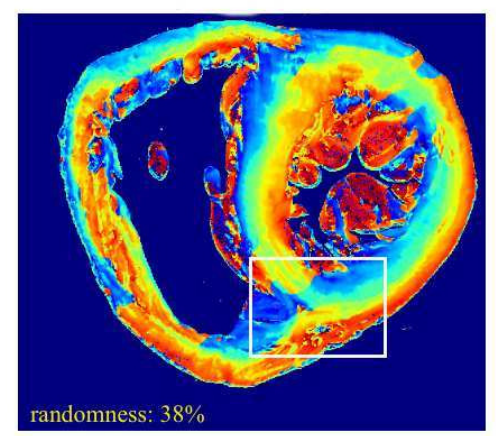

C

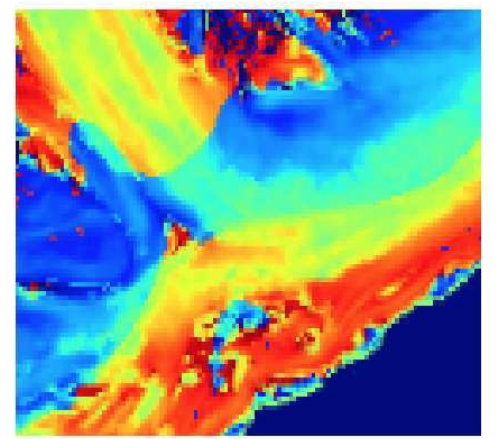

E

Fig. 4. Inclination angle maps of a coronal ventricular slice: (A) Absolute inclination. (B) and (D) Signed $\alpha$ from tilting measurements. (C) and (E) Signed $\alpha$ after TV restoration. The white rectangle represents the region of interest displayed in (D) and (E). The angle is coded in (reversed) grey scale $\left(0^{\circ}\right.$ to $\left.90^{\circ}\right)$ in (A) and in false color $\left(-90^{\circ}\right.$ to $\left.90^{\circ}\right)$ otherwise. The best results are achieved by the extended TV algorithm on these real data.

estimation of the signs with the tiltable stage is significantly ameliorated. Indeed, the image restoration process substantially reduces the noise and therefore provides a better estimation of the sign of $\alpha$ and consequently resolves the sign ambiguity.

Thanks to this elucidation of the inclination sign and the high-resolution of 3D-PLI, local information from individual voxels can be properly integrated to produce a more global insight into the whole fiber architecture of the heart and of the brain white matter.

\section{Acknowledgments}

This work was partly supported by ANR "MOSIFAH" under ANR-13-MONU-0009-01, the ERC under the European Union's Horizon 2020 research and innovation program (ERC Advanced Grant agreement No 694665:CoBCoM).

\section{REFERENCES}

[1] Markus Axer, Katrin Amunts, David Grässel, Christoph Palm, Jürgen Dammers, Hubertus Axer, Uwe Pietrzyk, and Karl Zilles, "A novel approach to the human connectome: ultra-high resolution mapping of fiber tracts in the brain," Neuroimage, vol. 54, no. 2, pp. 1091-1101, 2011.
[2] LA Pajdzik and AM Glazer, "Three-dimensional birefringence imaging with a microscope tilting-stage. i. uniaxial crystals," Journal of applied crystallography, vol. 39, no. 3, pp. 326-337, 2006.

[3] Markus Axer, David Graessel, Melanie Kleiner, Juergen Dammers, Timo Dickscheid, Julia Reckfort, Tim Huetz, Bjoern Eiben, Uwe Pietrzyk, Karl Zilles, et al., "Highresolution fiber tract reconstruction in the human brain by means of three-dimensional polarized light imaging," Frontiers in Neuroinformatics, vol. 5, pp. 34, 2011.

[4] Melanie Dohmen, Towards the Reconstruction of Fiber Tracts in the Human Brain by Means of $3 D$ Polarized Light Imaging, Ph.D. thesis, Universität Wuppertal, Fakultät für Mathematik und Naturwissenschaften» Physik» Dissertationen, 2014.

[5] Leonid I Rudin, Stanley Osher, and Emad Fatemi, "Nonlinear total variation based noise removal algorithms," Physica D: Nonlinear Phenomena, vol. 60, no. 1, pp. 259-268, 1992.

[6] Rachid Deriche and Olivier Faugeras, Les EDP en traitement des images et vision par ordinateur, Ph.D. thesis, INRIA, 1995. 